\title{
Treatment of external genital warts: a randomised clinical trial comparing podophyllin, cryotherapy, and electrodesiccation
}

\author{
K M Stone, T M Becker, A Hadgu, S J Kraus
}

\begin{abstract}
Four hundred and fifty patients were enrolled into a randomised clinical trial in a public sexually transmitted diseases clinic to evaluate the efficacy of podophyllin, cryotherapy, and electrodesiccation for treatment of external genital warts. Complete clearance of warts was observed in $41 \%, 79 \%$, and $94 \%$ of patients who received up to six weekly treatments of podophyllin, cryotherapy, and electrodesiccation, respectively. Relapses occurred in $25 \%$ of all patients, yielding 3 month clearance rates of $17 \%, 55 \%$, and $71 \%$ for podophyllin, cryotherapy, and electrodesiccation, respectively. Wart volume and duration did not influence treatment outcome. Response to therapy was greater in women than in men, and did not differ by treatment modality. Electrodesiccation and cryotherapy were more effective than podophyllin for the treatment of external genital warts, but none of these three treatments were highly successful.
\end{abstract}

Treatment of genital warts is often frustrating for clinicians and patients alike. ${ }^{1}$ Unlike single-dose therapy for many bacterial sexually transmitted diseases (STD), genital warts may require multiple applications of a local destructive therapy. Such therapy is often painful and sometimes unsuccessful. Moreover, genital warts commonly recur after "successful" therapy. Podophyllin has been the most common method of therapy for genital warts in public STD clinics because of its convenience and low cost, but low clearance rates have prompted greater interest in alternative forms of therapy. Previous clinical trials demonstrated clearance rates for podophyllin ranging from $22-77 \%^{2-5}$ and have shown that cryotherapy is superior to podophyllin. ${ }^{2}$ Electrosurgery and cryotherapy have been shown to produce clearance rates for genital warts ranging from $63-91 \%{ }^{245}$ Because no published studies have

Division of Sexually Transmitted Diseases Centers for Disease Control, Atlanta, Georgia, USA

$\mathrm{K} M$ Stone, $\mathrm{T} M$ Becker, $A$ Hadgu, $\mathrm{S}$ J Kraus compared podophyllin, cryotherapy, and electrosurgery, we conducted a randomised clinical trial using these three methods to treat genital warts in a public STD clinic in Atlanta, Georgia.

\section{Methods}

PATIENT ENROLMENT AND FOLLOW-UP

Between September 1984 and May 1986, all persons with external genital warts not treated in the previous month were candidates for this study. Exclusion criteria were: pregnancy; age less than 18 years; allergy to podophyllin, tincture of benzoin compound, or lidocaine; presence of obvious internal (cervical, vaginal or anal) warts or Buschke-Lowenstein lesions; or contraindications for electrosurgical procedures (such as presence of a cardiac pacemaker). Presence of exophytic genital warts was determined by visual macroscopic inspection only, without biopsy confirmation. Subclinical human papillomavirus infection was not assessed. Among eligible patients, 34 refused participation in the study, most often due to conflicts with work schedules. No monetary incentives for participation were offered. After written informed consent was obtained, patients were randomised to receive podophyllin, cryotherapy, or electrodesiccation according to a computer-generated randomisation schedule. Patients who missed a weekly visit or who were due for a post-therapy follow-up visit were contacted by telephone and/or letter. Patients were advised to refrain from sexual intercourse during therapy, and to refer their partners to the clinic for evaluation.

\section{TREATMENT REGIMENS}

All treatments were provided weekly, until all warts had cleared or until a total of six treatments had been administered. The maximum surface area treated with any method in a single visit was $4 \mathrm{~cm}^{2}$. Twentyfive percent podophyllin in tincture of benzoin compound was supplied by a local pharmacy. With care taken to avoid painting the surrounding skin, podophyllin was applied to the wart with a cotton swab. Patients were instructed to wash off the podophyllin 2 hours after the first treatment; this interval was lengthened by 2 hours to a maximum of 12 hours with each successive treatment, if the 
preceding application did not produce excessive irritation which the patient considered objectionable. Cryotherapy was administered using liquid nitrogen applied with a tapered cotton pledget on the end of a wooden applicator stick. The pledget was applied long enough to freeze the wart and a $1 \mathrm{~mm}$ margin of surrounding skin; each wart was frozen once.

Electrofulguration (a modification of electrodessication in which the electrode does not touch the tissue) was carried out with an electrosurgical apparatus (Bovie Specialist ${ }^{\mathrm{TM}}$ ) using a point electrode and standard settings for electrodesiccation, after local anaesthesia with subcutaneous $1 \%$ lidocaine. Patients were asked to return one week after each treatment. Patients whose genital warts cleared were asked to return in three months for follow-up evaluation, or sooner if warts reappeared. Patients whose warts did not clear after six applications of the assigned treatment were considered treatment failures and subsequently received the treatment modality of their choice.

\section{ANALYSIS}

We calculated a requisite study sample size of at least 450 patients ( 150 in each group) using an alpha error of 0.05 and power of 0.90 . Chi-square and Fisher's exact test were used for comparing differences in proportions; the $t$ test was used to compare means. Patients who failed to complete the treatment regimen or who did not return for a 3 month followup visit after wart clearance were considered noncompliant and were excluded from analysis. Although patients were clearly instructed and reminded to return in 3 months, many patients reported late. Wart-free patients who reported for follow-up later than 3 months but within 5 months after therapy were assumed to have been wart-free 3 months after therapy, and their visits have been considered as " 3 month" follow-up visits.

\section{Results}

STUDY POPULATION

We enrolled 450 patients; 144, 154, 152 received podophyllin, cryotherapy, and electrodesiccation, respectively (table 1). Despite frequent telephone follow-up efforts, all treatment groups had high rates of noncompliance. Compliance rates were higher for female, white, and older patients. Two hundred and thirty-seven patients completed the initial treatment regimen.

Patients in the podophyllin group were less likely to complete the regimen than those receiving cryotherapy or electrodesiccation $(44 \%, 56 \%$ and $58 \%$ respectively; $p=0.03$ ). Among 177 patients who completed therapy and whose warts cleared, 104 returned for a follow-up visit 3-5 months after wart clearance. Failure to report for follow-up was similar for all three treatment groups. Among the 164 compliant patients, the three treatment groups did not differ with respect to sex, age, race, marital status, sexual preference, or number of partners (table 2).

\section{CLEARANCE RATES}

Average total wart volume, average duration of warts prior to enrolment, and proportion of patients with anal warts were similar for all three treatment groups. Among the 237 patients who completed the treatment regimen, $177(75 \%)$ completely cleared their genital warts. Podophyllin, cryotherapy, and electrodesiccation resulted in wart clearance in 26/63 $(41 \%), 68 / 86(79 \%)$, and $83 / 88(94 \%)$ of patients, respectively (table 1). Electrodesiccation was more successful than cryotherapy $(p=0.003)$, which was more successful than podophyllin $(p<0.0001)$. At the three month follow-up visit, genital warts were present in $25 \%$ of patients; relapse rates were similar for all three treatment groups $(p=0 \cdot 17)$. To calculate efficacy of each method to produce persistent clearance of warts, initial treatment failures are included in the denominator along with relapses (table 3) to give clearance rates of $71 \%, 55 \%$, and $17 \%$ for electrodesiccation, cryotherapy, and podophyllin, respectively. The clearance rate for podophyllin is significantly lower than that for cryotherapy $(p<0.0001$ ) or electrodesiccation ( $p<$ 0.0001 ). Although the clearance rate for electrodesiccation was not significantly different from that of cryotherapy $(p=0.09)$, one clinical advantage was apparent. Fewer treatments with electrodesiccation

Table 1 Patient compliance and response to therapy of genital warts

\begin{tabular}{|c|c|c|c|c|}
\hline & Total & Podophyllin & Cryotherapy & Electrodesiccation \\
\hline $\begin{array}{l}\text { Number entered } \\
\text { Did not complete therapy } \\
\text { Completed therapy protocol } \\
\text { Warts persisted after six treatments } \\
\text { Wart-free within six treatments } \\
\text { Lost to follow up }{ }^{\star} \\
\text { Returned for } 3-5 \text { month follow up } \\
\text { Wart-free at follow up } \\
\text { Warts present at follow up }\end{array}$ & $\begin{array}{r}450 \\
213 \\
237 \\
60 \\
177 \\
73 \\
104 \\
78 \\
26\end{array}$ & $\begin{array}{r}144 \\
81 \\
63 \\
37 \\
26 \\
10 \\
16 \\
9 \\
7\end{array}$ & $\begin{array}{r}154 \\
68 \\
86 \\
18 \\
68 \\
26 \\
42 \\
33 \\
9\end{array}$ & $\begin{array}{r}152 \\
64 \\
88 \\
5 \\
83 \\
37 \\
46 \\
36 \\
10\end{array}$ \\
\hline
\end{tabular}

$\star$ Non-compliant patients. 
Table 2 Selected demographic and behavioural characteristics of study patients with genital warts

\begin{tabular}{|c|c|c|c|c|}
\hline \multirow[b]{2}{*}{ Characteristic } & \multirow[b]{2}{*}{$\begin{array}{l}\text { Non-compliant } \\
(N=266)\end{array}$} & \multicolumn{3}{|l|}{ Compliant } \\
\hline & & $\begin{array}{l}\text { Podophyllin } \\
(N=53)\end{array}$ & $\begin{array}{l}\text { Cryotherapy } \\
(N=60)\end{array}$ & $\begin{array}{l}\text { Electrodesiccation } \\
(N=51)\end{array}$ \\
\hline $\begin{array}{l}\% \text { Male } \\
\% \text { White } \\
\% \text { Single } \\
0 \text { Gay/bisexual } \\
\overline{\mathrm{X}} \text { age (years) } \\
\mathrm{X} \text { age at first intercourse (years) } \\
\overline{\mathrm{X}} \text { partners previous month } \\
\overline{\mathrm{X}} \text { partners previous six months }\end{array}$ & $\begin{array}{l}84^{\star} \\
46^{\star} \\
81 \\
19 \\
25 \cdot 5^{\star} \\
15 \cdot 2 \\
1 \cdot 3 \\
3 \cdot 8\end{array}$ & $\begin{array}{l}72 \\
62 \\
85 \\
21 \\
26 \cdot 7 \\
15 \cdot 9 \\
1 \cdot 1 \\
2 \cdot 9\end{array}$ & $\begin{array}{l}72 \\
52 \\
85 \\
21 \\
27 \cdot 3 \\
15 \cdot 9 \\
1 \cdot 1 \\
4 \cdot 2\end{array}$ & $\begin{array}{l}75 \\
57 \\
80 \\
26 \\
26 \cdot 9 \\
15 \cdot 5 \\
1 \cdot 4 \\
3 \cdot 8\end{array}$ \\
\hline
\end{tabular}

$\star$ Non-compliant $v s$ compliant, $\mathrm{p}<0.03$.

(mean, 1.3) were required to achieve complete clearance of warts compared with the number required with cryotherapy (3.2) or podophyllin (3.4). Post-treatment pain was reported by similar proportions $(17 \%)$ of patients in each treatment group. Local infection was observed in one patient after cryotherapy and none of the patients treated with podophyllin or electrodesiccation.

Genital wart clearance rates did not differ significantly by total wart volume, duration prior to therapy, or anatomic site (table 3). Among patients treated with podophyllin, wart clearance was somewhat less likely in persons whose genital warts were present more than 30 days prior to therapy, compared with those with warts of shorter duration $(3 / 32$ vs $6 / 21 ; p=0.07)$. In women, clearance rates were higher than in males (table 3 ), and efficacy was similar for all three treatments (podophyllin: $7 / 15$ (47\%), cryotherapy: $11 / 17(65 \%)$, electrodesiccation: $11 / 13(85 \%) ; p=0.6)$. Wart volume and duration prior to therapy were similar for males and

Table 3 Clearance rates ${ }^{\star}$ among compliant patients with genital warts



«Number wart-free at 3-5 months follow-up/number of compliant patients. females. In men, the clearance rate for gay/bisexual men was lower than, but not significantly different from, the clearance rate in heterosexual men $(9 / 27 \mathrm{vs}$ $39 / 91 ; p=0.4)$.

\section{Discussion}

This study confirms previous observations that cryotherapy and electrocautery produce higher clearance rates than podophyllin for external genital warts, and that none of these methods are highly successful. Some previous studies reported that response to genital wart therapy is influenced by individual wart area, ${ }^{67}$ total wart area, ${ }^{78}$ duration, ${ }^{68}$ and anatomic site. ${ }^{9}$ In our study, individual wart size was not addressed, and total cubic volume did not affect clearance rates in any of the treatment groups. Only in patients treated with podophyllin did the duration of warts appear to affect clearance rates, and differences were not large. However, our sample size is too small to detect a significant difference. Newer warts may be more amenable to treatment than older warts because they are less heavily keratinised. Keratinisation would be expected to impede local therapy with podophyllin, but not with more destructive methods such as cryotherapy or electrodesiccation. Anal warts were no more resistant to therapy than genital warts in our study; however, because only seven evaluable patients had anal warts, the statistical power to detect a significant difference is low. Although some studies have found that anal warts are more difficult to treat than genital warts, ${ }^{9}$ such differences may be related to host factors rather than to differences in anal epithelium. In women, anal warts are no more resistant to treatment than genital warts. ${ }^{9}$ In contrast, anal warts in gay men may be a marker for immunosuppression, ${ }^{10}$ and a recent study found that HIV-induced immunosuppression was clearly related to lower clearance rates for anogenital warts. ${ }^{.1}$

The higher clearance rate in females may relate to higher rates of spontaneous regression ${ }^{7}$ or greater ease of eradication of warts in nonkeratinised areas, 
compared to keratinised sites. Earlier presentation to the clinic, when warts were smaller and fewer in number, does not explain this difference, since wart volume and duration were similar for males and females.

This study clearly illustrates the poor compliance with therapy which is common among public STD clinic populations. Low compliance provides a potential source of bias in our study results; however, failure to complete the therapy regimen and losses to follow up were high in all treatment groups. Monetary incentives might have improved compliance, but would have limited the generalisability of our findings. Factors which may affect treatment outcome and recurrence but were not assessed in our study include concurrent HIV infection, presence of warts in the anal canal, adjacent subclinical human papillomavirus infection, and exposure to partners with warts. Misdiagnosis of other epithelial conditions (pearly penile papules, molluscum contagiosum, fibroepithelial polyps, heterotopic sebaceous glands) as genital warts is a potential source of misclassification. However, the randomised design of this study should have minimised the likelihood of bias due to any of these factors.

Public STD program managers and clinic staff, as well as private physicians, should evaluate the feasibility of offering cryotherapy or electrodesiccation to treat genital warts. A comparison of costs and benefits of cryotherapy or electrocautery might show them to be less expensive than podophyllin, since fewer clinic visits are required. Other than a liquid nitrogen storage tank which must be refilled periodically, the equipment required for cryotherapy is fairly simple: an insulated dispensing container (such as a Thermos ${ }^{\mathrm{TM}}$ ) and cotton-tipped wooden sticks. Electrodesiccation is more complicated and time-consuming, since it requires a special apparatus, injection of local anaesthesia, and careful positioning of the patient on a ground pad. Initial outlay for electrodesiccation equipment is greater than for cryotherapy; however, recurring costs are minimal. Cryotherapy and electrocautery both are considered surgical procedures, and should be performed only by personnel who are adequately trained and supervised. In some jurisdictions, malpractice policies may not allow such procedures to be performed by nurses. Many clinicians and patients welcome alternative therapies to podophyllin for genital warts. Podophyllotoxin (recently renamed podofilox), one of the active ingredients in crude podophyllin resin, is being evaluated in the U.S. and elsewhere as an alternative to podophyllin. Efficacy appears similar to that of podophyllin. However, podophyllotoxin offers many advantages over the crude resin: less local toxicity, purity, stable shelf life, and negligible systemic absorption. ${ }^{12}$ Recently, interferon was licensed for intralesional therapy of exter- nal genital warts. ${ }^{13}$ However, success with interferon has been limited, ${ }^{6-814} 15$ and its expense will preclude widespread use. Cryotherapy and electrodesiccation should be considered valuable additions to our armanentarium of locally destructive methods to eradicate external genital warts.

TM Use of trademark is for identification purposes only and does not imply endorsement by the Department of Health and Human Services.

The authors are grateful to Kathy Heidish, R.N., Rick Miller, Linda Hedlund, M.D., and John Long, M.D., for their participation in this study; Wil Whittington, E Russell Alexander, M.D., and Robert Johnson, M.D., for manuscript review; Richard Brautigan for inspiration; and Ms Ruby Booth for assistance in manuscript preparation.

\section{Statistical reviewer: Alula Hadgu.}

Address for correspondence: Katherine $M$ Stone, MD, Centers for Disease Control, Division of Sexually Transmitted Diseases, Mail Stop E02, Atlanta, GA 30333, USA.

Address for reprints: Technical Information Services, Centers for Disease Control, Centre for Prevention Services, Mail Stop E06, Atlanta, GA 30333, USA.

1 Margolis S. Therapy for condyloma acuminatum. Rev Inf Dis 1982;4(suppl):S829-S35.

2 Bashi SA. Cryotherapy versus podophyllin in the treatment of genital warts. Int J Dermatol 1985;24:535-6.

3 Jensen SL. Comparison of podophyllin application with simple surgical excision in clearance and recurrence of perianal condylomata acuminata. Lancet $1985 ; \mathrm{ii}: 1146-8$.

4 Simmons PD. Podophyllin $10 \%$ and $25 \%$ in the treatment of ano-genital warts. $\mathrm{Br} J$ Venereal Dis 1981;57:208-9.

5 Simmons PD, Langlet F, Thin RNT. Cryotherapy versus electrocautery in the treatment of genital warts. $B r J$ Venereal Dis 1981;57:273-4.

6 Eron LJ, Judson F, Tucker S, et al. Interferon therapy for condylomata acuminata. $N$ Engl J Med 1986;315:1059-64.

7 Keay S, Teng N, Eisenberg M, Story B, Sellers PW, Merigan TC. Topical interferon for treating condyloma acuminata in women. J Infect Dis 1988;158:934-9.

8 Friedman-Kien AE, Eron LJ, Conant $M$, et al. Natural interferon alfa for treatment of condylomata acuminata. JAMA 1988;259:533-8.

9 Balsdon MJ. Cryosurgery of genital warts [Letter]. Br J Venereal Dis 1978;54:352-3.

10 Kent C, Samuel M, Winkelstein W. The role of anal/genital warts in HIV infection [Letter]. JAMA 1987;258:3385-6.

11 Douglas JM, Rogers M, Judson FN. The effect of asymptomatic infection with HTLV-III on the response of anogenital warts to intralesional treatment with recombinant $a_{2}$ interferon. $J$ Infect Dis 1986;154:331-4.

12 Beutner KR. Podophyllin in the treatment of genital human papillomavirus infection: a review. Sem Dermatol 1987;6: 10-8.

13 Food and Drug Administration. Alpha interferon for venereal warts. FDA Drug Bulletin 1988;18:19-20.

14 Kirby $P$. Interferon and genital warts: much potential, modest progress (editorial). JAMA 1988;259:570-2.

15 Reichman RC, Oakes D, Bonnez W, et al. Treatment of condyloma acuminatum with three different interferons administered intralesionally: A double-blind, placebo-controlled trial. Ann Intern Med 1988;108:675-9.

Accepted for publication 8 September 1989 